\title{
New result on fixed point theorems for $\varphi$-contractions in Menger spaces
}

\author{
Huichun Hua ${ }^{1}$, Minjiang Chen ${ }^{2}$ and Shenghua Wang ${ }^{1 *}$
}

"Correspondence:

sheng-huawang@hotmail.com

${ }^{1}$ Department of Mathematics and

Physics, North China Electric Power

University, Baoding, 071003, China

Full list of author information is

available at the end of the article

\section{Springer}

\begin{abstract}
Very recently, Fang (Fuzzy Sets Syst. 267:86-99, 2015) gave some fixed point theorems for probabilistic $\varphi$-contractions in Menger spaces. Fang's results improve the one of Jachymski (Nonlinear Anal. 73:2199-2203, 2010) by relaxing the restriction on the gauge function $\varphi$. In this paper, inspired by the results of Fang, we prove a new fixed point theorem for a probabilistic $\varphi$-contraction in Menger spaces in which a weaker condition on the function $\varphi$ is required. Our result improves the corresponding one of Fang and some others. Finally, an example is given to illustrate our result.
\end{abstract}

MSC: $54 \mathrm{E} 70 ; 47 \mathrm{H} 25$

Keywords: Menger metric space; probabilistic $\varphi$-contraction; Cauchy sequence; fixed point theorem

\section{Introduction}

Let $(X, F, \Delta)$ be a probabilistic metric space and $T: X \rightarrow X$ be a mapping. If there exists a gauge function $\varphi: \mathbb{R}^{+} \rightarrow \mathbb{R}^{+}$such that

$$
F_{T x, T y}(\varphi(t)) \geq F_{x, y}(t) \quad \text { for all } x, y \in X \text { and } t>0,
$$

then the mapping $T$ is called a probabilistic $\varphi$-contraction. The probabilistic $\varphi$-contraction is a generalization of probabilistic $k$-contraction given by Sehgal and Bharucha-Reid [1]. In literature, many authors investigated fixed point theorems for probabilistic $\varphi$-contractions in Menger spaces; see [2-7]. On the fixed point theorems for other types of contractions in Menger or fuzzy metric spaces, please see [8-12]. Recently, Jachymski [13] proved a new fixed point theorem for a probabilistic $\varphi$-contraction in which the condition on the function $\varphi$ is weakened. More precisely, the author gave the following result.

Theorem 1.1 ([13]) Let $(X, F, \Delta)$ be a complete Menger probabilistic metric space with a continuous t-norm $\Delta$ of H-type, and let $\varphi: \mathbb{R}^{+} \rightarrow \mathbb{R}^{+}$be a function satisfying conditions:

$$
0<\varphi(t)<t \quad \text { and } \quad \lim _{n \rightarrow \infty} \varphi^{n}(t)=0 \quad \text { for all } t>0 .
$$

If $T: X \rightarrow X$ is a probabilistic $\varphi$-contraction, then $T$ has a unique fixed point $x^{*} \in X$, and $\left\{T^{n} x_{0}\right\}$ converges to $x^{*}$ for each $x_{0} \in X$.

(c) 2015 Hua et al. This article is distributed under the terms of the Creative Commons Attribution 4.0 International License (http://creativecommons.org/licenses/by/4.0/), which permits unrestricted use, distribution, and reproduction in any medium, provided you give appropriate credit to the original author(s) and the source, provide a link to the Creative Commons license, and indicate if changes were made. 
Although Theorem 1.1 has been a very perfect result in which the condition on the gauge function $\varphi$ is very simple, Fang [14] improves Theorem 1.1 by giving a new condition on $\varphi$ recently. Let $\varphi: \mathbb{R}^{+} \rightarrow \mathbb{R}^{+}$be a function satisfying the following condition:

$$
\text { for each } t>0 \text { there exists } r \geq t \text { such that } \lim _{n \rightarrow \infty} \varphi^{n}(t)=0 \text {. }
$$

Let $\boldsymbol{\Phi}_{\mathbf{w}}$ denote the set of all functions $\varphi: \mathbb{R}^{+} \rightarrow \mathbb{R}^{+}$satisfying the condition (1.1) and let $\boldsymbol{\Phi}$ denote the set of all functions $\varphi: \mathbb{R}^{+} \rightarrow \mathbb{R}^{+}$satisfying the condition that $\lim _{n \rightarrow \infty} \varphi^{n}(t)=0$ for all $t>0$. In [14], Fang gave an example of $\varphi \in \boldsymbol{\Phi}_{\mathbf{w}}$ but $\varphi \notin \boldsymbol{\Phi}$.

By using the condition (1.1), Fang gave the following result.

Theorem 1.2 ([14]) Let $(X, F, \Delta)$ be a complete Menger space with a t-norm $\Delta$ of H-type. If $T: X \rightarrow X$ is a probabilistic $\varphi$-contraction, where $\varphi \in \boldsymbol{\Phi}_{\mathbf{w}}$, then $T$ has a unique fixed point $x^{*} \in X$, and $\left\{T^{n} x_{0}\right\}$ converges to $x^{*}$ for each $x_{0} \in X$.

Since the condition (1.1) is weaker than the one in Theorem 1.1, Theorem 1.2 improves Theorem 1.1. In [14], Fang asked the following question:

Can the condition (1.1) in Theorem 1.2 be replaced by a more weak condition?

In this paper, we give a positive answer to the question of Fang by proving a new fixed point theorem for a probabilistic $\varphi$-contraction in Menger spaces. In our result, the function $\varphi$ is required to satisfy a more weak condition than (1.1) and the $t$-norm is not required to be of $H$-type. Our result improves the corresponding one of Fang [14] and some others. Finally, an example is given to illustrate our result.

\section{Preliminaries}

In the rest of this paper, let $\mathbb{R}=(-\infty,+\infty), \mathbb{R}^{+}=[0,+\infty)$ and $\mathbb{N}$ denote the set of all natural numbers.

A mapping $F: \mathbb{R} \rightarrow[0,1]$ is called a distribution function if it is non-decreasing and left-continuous with $\inf _{t \in \mathbb{R}} F(t)=0$. If in addition $F(0)=0$, then $F$ is called a distance distribution function. A distance distribution function $F$ satisfying $\lim _{t \rightarrow \infty} F(t)=1$ is called a Menger distance distribution function.

The set of all Menger distance distribution functions is denoted by $\mathcal{D}^{+}$. It is known that $\mathcal{D}^{+}$is partially ordered by the usual pointwise ordering of functions, that is, $F \leq G$ if and only if $F(t) \leq G(t)$ for all $t \geq 0$. The maximal element in $\mathcal{D}^{+}$on this order is the distance distribution function $\epsilon_{0}$ defined by

$$
\epsilon_{0}(t)= \begin{cases}0, & t=0 \\ 1, & t>0 .\end{cases}
$$

Definition 2.1 ([15]) A binary operation $\Delta:[0,1] \times[0,1] \rightarrow[0,1]$ is a $t$-norm if $\Delta$ satisfies the following conditions:

(1) $\Delta$ is associative and commutative;

(2) $\Delta(a, 1)=a$ for all $a \in[0,1]$;

(3) $\Delta(a, b) \leq \Delta(c, d)$ whenever $a \leq c$ and $b \leq d$ for all $a, b, c, d \in[0,1]$.

Two typical examples of the continuous $t$-norm are $\Delta_{P}(a, b)=a b$ and $\Delta_{M}(a, b)=$ $\min \{a, b\}$ for all $a, b \in[0,1]$. 
Definition 2.2 ([16]) A $t$-norm $\Delta$ is said to be of Hadžić-type (for short $H$-type) if the family of functions $\left\{\Delta^{m}(t)\right\}_{m=1}^{\infty}$ is equicontinuous at $t=1$, where

$$
\Delta^{1}(t)=\Delta(t, t), \quad \Delta^{m+1}(t)=\Delta\left(t, \Delta^{m}(t)\right), \quad m=1,2, \ldots, t \in[0,1]
$$

It is easy to see that $\Delta_{M}$ is a $t$-norm of $H$-type but $\Delta_{P}$ is not of $H$-type. Here we give a new $t$-norm of $H$-type by $\Delta_{M}$ and $\Delta_{P}$.

Example 2.1 Let $\Delta(x, 1)=\Delta(1, x)=x$ for all $x \in[0,1], \Delta(x, y)=\Delta_{P}(x, y)$ for all $x, y \in[0,1]$ with $\max \{x, y\} \in\left[0, \frac{1}{2}\right]$ and $\Delta(x, y)=\Delta_{M}(x, y)$ for all $x, y \in[0,1]$ with $\max \{x, y\} \in\left(\frac{1}{2}, 1\right]$. It is easy to check that $\Delta$ is a $t$-norm. Now we show that it is of $H$-type. For any given $\epsilon \in\left(0, \frac{1}{2}\right)$, set $\delta=\epsilon$. Then $1-\delta=1-\epsilon>\frac{1}{2}$. Thus, for all $t \in(1-\delta, 1)$, one has $\Delta^{n}(t)=t>1-\delta=1-\epsilon$ for all $n \in \mathbb{N}$. For $\epsilon \in\left[\frac{1}{2}, 1\right)$, taking $\delta \in\left(0, \frac{1}{2}\right)$ arbitrarily, then we have $1-\delta>\frac{1}{2} \geq 1-\epsilon$. Thus for all $t \in(1-\delta, 1), \Delta^{n}(t)=t>1-\delta>\frac{1}{2} \geq 1-\epsilon$ for all $n \in \mathbb{N}$. Therefore, $\Delta$ is a $t$-norm of $H$-type.

Example 2.2 Let $\delta \in(0,1]$ and let $\Delta$ be a $t$-norm. Define $\Delta_{\delta}$ by $\Delta_{\delta}(x, y)=\Delta(x, y)$, if $\max \{x, y\} \leq 1-\delta$, and $\Delta_{\delta}(x, y)=\min \{x, y\}$, if $\max \{x, y\}>1-\delta$. then $\Delta_{\delta}$ is a $t$-norm of $H$-type; see [17]. However, if $\Delta_{\delta}(x, 1)=\Delta_{\delta}(1, x)=x$ for all $x \in[0,1], \Delta_{\delta}(x, y)=\delta$ for all $x, y \in[\delta, 1)$ and $\Delta_{\delta}(x, y)=0$ for all $x, y \in[0,1]$ with $\min \{x, y\} \in[0, \delta)$, then $\Delta_{\delta}$ is a $t$-norm but not of $H$-type.

For other $t$-norms of $H$-type, the reader may refer to [16].

Definition 2.3 ([18]) A triple $(X, F, \Delta)$ is called a Menger probabilistic metric space (for short, Menger space) if $X$ is a nonempty set, $\Delta$ is a $t$-norm, and $F$ is a mapping from $X \times X \rightarrow \mathcal{D}^{+}$satisfying the following conditions (for $x, y \in X$, denote $F(x, y)$ by $F_{x, y}$ ):

(PM-1) $F_{x, y}(t)=\epsilon_{0}(t)$ for all $t \in \mathbb{R}$ if and only if $x=y$;

(PM-2) $F_{x, y}(t)=F_{y, x}(t)$ for all $t \in \mathbb{R}$;

(PM-3) $F_{x, y}(t+s) \geq \Delta\left(F_{x, z}(t), F_{z, y}(s)\right)$ for all $x, y, z \in X$ and $t, s>0$.

Definition 2.4 ([15]) Let $(X, F, \Delta)$ be a Menger space and $\left\{x_{n}\right\}$ be a sequence in $X$. The sequence $\left\{x_{n}\right\}$ is said to be convergent to $x \in X$ if $\lim _{n \rightarrow \infty} F_{x_{n}, x}(t)=1$ for all $t>0$; the sequence $\left\{x_{n}\right\}$ is said to be a Cauchy sequence if for any given $t>0$ and $\epsilon \in(0,1)$, there exists $N_{\epsilon, t} \in \mathbb{N}$ such that $F_{x_{n}, x_{m}}(t)>1-\epsilon$ whenever $m, n>N_{t, \epsilon}$; the Menger space $(X, F, \Delta)$ is said to be complete, if each Cauchy sequence in $X$ is convergent to some point in $X$.

\section{Main results}

In this section, let $\boldsymbol{\Phi}_{\mathbf{w}^{*}}$ denote the set of all functions $\varphi: \mathbb{R}^{+} \rightarrow \mathbb{R}^{+}$satisfying the following condition:

for each $t_{1}, t_{2}>0$ there exists $r \geq \max \left\{t_{1}, t_{2}\right\}$ and $N \in \mathbb{N}$

$$
\text { such that } \varphi^{n}(r)<\min \left\{t_{1}, t_{2}\right\} \text { for all } n>N \text {. }
$$

Obviously, the condition (3.1) implies that

for each $t>0$ there exists $r \geq t$ and $N \in \mathbb{N}$

such that $\varphi^{n}(r)<t$ for all $n>N$. 
It is easy to see that for each $\varphi \in \boldsymbol{\Phi}_{\mathbf{w}}, \varphi \in \boldsymbol{\Phi}_{\mathbf{w}^{*}}$. In fact, if $\varphi \in \boldsymbol{\Phi}_{\mathbf{w}}$, then for each $t_{1}, t_{2}>0$, there exist $r_{1} \geq t_{1}$ and $r_{2} \geq t_{2}$ such that $\lim _{n \rightarrow \infty} \varphi^{n}\left(r_{1}\right)=\lim _{n \rightarrow \infty} \varphi^{n}\left(r_{2}\right)=0$. Assume that $t_{1} \leq t_{2}$. Then there exists $N \in \mathbb{N}$ such that $\varphi^{n}\left(r_{2}\right)<t_{1}$ for all $n>N$. Thus $\varphi \in \boldsymbol{\Phi}_{\mathbf{w}^{*}}$.

However, if $\varphi \in \boldsymbol{\Phi}_{\mathbf{w}^{*}}$, then it is unnecessary that $\varphi \in \boldsymbol{\Phi}_{\mathbf{w}}$.

Example 3.1 Let $\varphi: \mathbb{R}^{+} \rightarrow \mathbb{R}^{+}$by $\varphi(t)=t$ for all $t \in[0,1], \varphi(t)=t-1$ for all $t \in(1, \infty)$. Then $\varphi \in \boldsymbol{\Phi}_{\mathbf{w}^{*}}$. In fact, for each $t_{1}, t_{2} \in(0, \infty)$, there exists $N \in \mathbb{N}$ such that $r=1+N+\epsilon>$ $\max \left\{t_{1}, t_{2}\right\}$, where $\epsilon \in\left(0, \min \left\{t_{1}, t_{2}, 1\right\}\right)$. Then we have $\varphi^{n}(r)=\epsilon<\min \left\{t_{1}, t_{2}\right\}$ for all $n>N+1$. So $\varphi \in \boldsymbol{\Phi}_{\mathbf{w}^{*}}$. However, since $\lim _{n \rightarrow \infty} \varphi^{n}(r) \neq 0$ for all $r>0, \varphi \notin \boldsymbol{\Phi}_{\mathbf{w}}$.

From Example 3.1 we see that $\boldsymbol{\Phi}_{\mathbf{w}^{*}}$ is a proper subclass of $\boldsymbol{\Phi}_{\mathbf{w}}$. On $\boldsymbol{\Phi}_{\mathbf{w}^{*}}, \boldsymbol{\Phi}_{\mathbf{w}}$, and $\boldsymbol{\Phi}$, we have $\boldsymbol{\Phi} \subset \boldsymbol{\Phi}_{\mathbf{w}} \subset \boldsymbol{\Phi}_{\mathbf{w}^{*}}$.

Lemma 3.1 Let $\varphi \in \boldsymbol{\Phi}_{\mathbf{w}^{*}}$. Then for each $t>0$, there exists $r \geq t$ such that $\varphi(r)<t$.

Proof Suppose that there is $t_{0}>0$ such that $\varphi(r) \geq t_{0}$ for all $r \geq t_{0}$. By induction, we obtain $\varphi^{n}(r) \geq t_{0}$ for all $n \in \mathbb{N}$. From (3.2) it follows that there exist $r \geq t_{0}$ and $N \in \mathbb{N}$ such that $\varphi^{n}(r)<t_{0}$ for all $n>N$, which contradicts $\varphi^{n}(r) \geq t_{0}$ for all $r \geq t_{0}$ and $n \in \mathbb{N}$. Thus for each $t>0$, there exists $r \geq t$ such that $\varphi(r) \leq t$. This completes the proof.

Lemma 3.2 Let $(X, F, \Delta)$ be a Menger space and $x, y \in X$. If there exists a function $\varphi \in \mathbf{\Phi}_{\mathbf{w}^{*}}$ such that

$$
F_{x, y}(\varphi(t)) \geq F_{x, y}(t), \quad \forall t>0,
$$

then $x=y$.

Proof First by a similar proof with Lemma 2.2 of [14] we can show that for all $n \in \mathbb{N}$ and $t>0$, one has $\varphi^{n}(t)>0$. By induction, from (3.3) it follows that

$$
F_{x, y}\left(\varphi^{n}(t)\right) \geq F_{x, y}(t) \quad \text { for all } n \in \mathbb{N} \text { and } t>0
$$

Next we show that $F_{x, y}(t)=1$ for all $t>0$. In fact, if there exists $t_{0}>0$ such that $F_{x, y}\left(t_{0}\right)<1$, then since $\lim _{t \rightarrow \infty} F_{x, y}(t)=1$ there is $t_{1}>t_{0}$ such that

$$
F_{x, y}(t)>F_{x, y}\left(t_{0}\right) \quad \text { for all } t \geq t_{1}
$$

Since $\varphi \in \boldsymbol{\Phi}_{\mathbf{w}^{*}}$, there exist $t_{2} \geq \max \left\{t_{1}, t_{0}\right\}$ and $N \in \mathbb{N}$ such that $\varphi^{n}\left(t_{2}\right)<\min \left\{t_{0}, t_{1}\right\}$ for all $n>N$. By the monotonicity of $F_{x, y}(\cdot)$, from (3.4) and (3.5) it follows that, for each $n>N$,

$$
F_{x, y}\left(t_{0}\right) \geq F_{x, y}\left(\varphi^{n}\left(t_{2}\right)\right) \geq F_{x, y}\left(t_{2}\right) \geq F_{x, y}\left(t_{1}\right)>F_{x, y}\left(t_{0}\right)
$$

It is a contradiction. Therefore, $F_{x, y}(t)=1$ for all $t>0$, i.e., $x=y$. This completes the proof.

Lemma 3.3 Let $(X, F, \Delta)$ be a Menger space where $\Delta$ is continuous at $(1,1)$ and let $\left\{x_{n}\right\}$ be a sequence in $X$. Suppose that there exists a function $\varphi \in \boldsymbol{\Phi}_{\mathbf{w}^{*}}$ satisfying the following conditions: 
(1) $\varphi(t)>0$ for all $t>0$;

(2) $F_{x_{n}, x_{m}}(\varphi(t)) \geq F_{x_{n-1}, x_{m-1}}(t)$ for all $n, m \in \mathbb{N}$ and $t>0$.

Then $\lim _{n \rightarrow \infty} F_{x_{n}, x_{n+k}}(t)=1$ for all $k \in \mathbb{N}$ and $t>0$.

Proof It is easy to see that the condition (1) implies that $\varphi^{n}(t)>0$ for all $t>0$ and the condition (2) implies that

$$
F_{x_{n}, x_{n+1}}\left(\varphi^{n}(t)\right) \geq F_{x_{0}, x_{1}}(t), \quad \forall n \in \mathbb{N} \text { and } \forall t>0 .
$$

We first prove that

$$
\lim _{n \rightarrow \infty} F_{x_{n}, x_{n+1}}(t)=1, \quad \forall t>0 .
$$

Since $\lim _{t \rightarrow \infty} F_{x_{0}, x_{1}}(t)=1$, for any $\epsilon \in(0,1)$, there exists $t_{0}>0$ such that $F_{x_{0}, x_{1}}\left(t_{0}\right)>1-\epsilon$. For each $t>0$, since $\varphi \in \boldsymbol{\Phi}_{\mathbf{w}^{*}}$, there exist $t_{1} \geq \max \left\{t, t_{0}\right\}$ and $N \in \mathbb{N}$ such that $\varphi^{n}\left(t_{1}\right)<\min \left\{t, t_{0}\right\}$ for all $n \geq N$. By the monotonicity of $F_{x, y}(\cdot)$, from (3.6) we have

$$
\begin{aligned}
F_{x_{n}, x_{n+1}}(t) & \geq F_{x_{n}, x_{n+1}}\left(\varphi^{n}\left(t_{1}\right)\right) \\
& \geq F_{x_{0}, x_{1}}\left(t_{1}\right) \geq F_{x_{0}, x_{n}}\left(t_{0}\right) \\
& >1-\epsilon \text { for all } n \geq N,
\end{aligned}
$$

which implies that (3.7) holds. Assume that $\lim _{n \rightarrow \infty} F_{x_{n}, x_{n+k}}(t)=1$ for each $k \in \mathbb{N}$ and $t>0$. Since $\Delta$ is continuous at $(1,1)$, we have

$$
F_{x_{n}, x_{n+k+1}}(t) \geq \Delta\left(F_{x_{n}, x_{n+k}}(t / 2), F_{x_{n+k}, x_{n+k+1}}(t / 2)\right) \rightarrow \Delta(1,1)=1 \quad \text { as } n \rightarrow \infty .
$$

By induction we conclude that

$$
\lim _{n \rightarrow \infty} F_{x_{n}, x_{n+k}}(t)=1, \quad \forall k \in \mathbb{N} \text { and } \forall t>0 .
$$

This completes the proof.

Lemma 3.4 Let $(X, F, \Delta)$ be a Menger space where $\Delta$ is of H-type and continuous at $(1,1)$ and let $\left\{x_{n}\right\}$ be a sequence in $X$. Suppose that there exists a function $\varphi \in \boldsymbol{\Phi}_{\mathbf{w}^{*}}$ satisfying the conditions (1) and (2) in Lemma 3.3. Then $\left\{x_{n}\right\}$ is a Cauchy sequence.

Proof Let $t>0$. By Lemma 3.1 there is $r \geq t$ such that $\varphi(r)<t$. We show by induction that

$$
F_{x_{n}, x_{n+k}}(t) \geq \Delta^{k}\left(F_{x_{n}, x_{n+1}}(t-\varphi(r))\right), \quad \forall k \in \mathbb{N} .
$$

Obviously, (3.8) holds for $k=1$. Assume that (3.8) holds for some $k \in \mathbb{N}$. By (2) in Lemma 3.3 we have

$$
\begin{aligned}
F_{x_{n}, x_{n+k+1}}(t) & \geq \Delta\left(F_{x_{n}, x_{n+1}}(t-\varphi(r)), F_{x_{n+1}, x_{n+k+1}}(\varphi(r))\right) \\
& \geq \Delta\left(F_{x_{n}, x_{n+1}}(t-\varphi(r)), F_{x_{n}, x_{n+k}}(r)\right)
\end{aligned}
$$




$$
\begin{aligned}
& \geq \Delta\left(F_{x_{n}, x_{n+1}}(t-\varphi(r)), F_{x_{n}, x_{n+k}}(t)\right) \\
& \geq \Delta\left(F_{x_{n}, x_{n+1}}(t-\varphi(r)), \Delta^{k}\left(F_{x_{n}, x_{n+1}}(t-\varphi(r))\right)\right) \\
& =\Delta^{k+1}\left(F_{x_{n}, x_{n+1}}(t-\varphi(r))\right) .
\end{aligned}
$$

It follows that (3.8) holds for $k+1$. So (3.8) holds for all $k \in \mathbb{N}$.

Let $t>0$. Define $a_{n}=\inf _{k \geq 1} F_{x_{n}, x_{n+k}}(t)$. Since $\varphi \in \boldsymbol{\Phi}_{\mathbf{w}^{*}}$, by Lemma 3.1 there exists $t_{0} \geq t$ such that $\varphi\left(t_{0}\right)<t$. So by the condition (2) we have

$$
\begin{aligned}
a_{n} & =\inf _{k \geq 1} F_{x_{n}, x_{n+k}}(t) \\
& \geq \inf _{k \geq 1} F_{x_{n}, x_{n+k}}\left(\varphi\left(t_{0}\right)\right) \\
& \geq \inf _{k \geq 1} F_{x_{n-1}, x_{n-1+k}}\left(t_{0}\right) \\
& \geq \inf _{k \geq 1} F_{x_{n-1}, x_{n-1+k}}(t) \\
& =a_{n-1} \quad \text { for all } n \in \mathbb{N} .
\end{aligned}
$$

So $\left\{a_{n}\right\}$ is non-decreasing. Since $\left\{a_{n}\right\}$ is bounded, there exists $a \in[0,1]$ such that $a_{n} \rightarrow a$ as $n \rightarrow \infty$. Assume that $a<1$. Then there exists $\eta \in(0,1)$ such that $a+\eta<1$. For any given $\epsilon \in(0,1 / 2)$, by the definition of $a_{n}$ there exists $k=k(\epsilon, n) \in \mathbb{N}$ such that

$$
a_{n} \geq F_{x_{n}, x_{n+k}}(t)-\epsilon / 2
$$

By Lemma 3.3 one has $\lim _{n \rightarrow \infty} F_{x_{n}, x_{n+1}}(t-\varphi(r))=1$. Therefore there exist $\delta \in(0,1)$ and $N \in \mathbb{N}$ such that $F_{x_{n}, x_{n+1}}(t-\varphi(r)) \in(1-\delta, 1)$ for all $n>N$. Since $\Delta$ is of $H$-type, $\Delta^{k}\left(F_{x_{n}, x_{n+1}}(t-\right.$ $\varphi(r)))>1-\epsilon / 2$ for all $n>N$ and all $k \in \mathbb{N}$. Further combing (3.8) and (3.9) we get

$$
1>a+\eta>a_{n} \geq 1-\epsilon
$$

for all $n>N$, which implies that

$$
1>a+\delta>a \geq 1
$$

It is a contradiction. So $a=1$. Since $a_{n} \rightarrow 1$ as $n \rightarrow \infty$, there exists $N^{\prime} \in \mathbb{N}$ such that $a_{n}>$ $1-\epsilon$ for all $n>N$. Then by the definition of $\left\{a_{n}\right\}$, we have

$$
F_{x_{n}, x_{n}+k}(t)>1-\epsilon
$$

for all $n \in \mathbb{N}$ with $n>N^{\prime}$ and all $k \in \mathbb{N}$. Thus $\left\{x_{n}\right\}$ is a Cauchy sequence. This completes the proof.

Theorem 3.1 Let $(X, F, \Delta)$ be a complete Menger space where $\Delta$ is of H-type and continuous at $(1,1)$. Let $T: X \rightarrow X$ be a probabilistic $\varphi$-contraction, where $\varphi \in \boldsymbol{\Phi}_{\mathbf{w}^{*}}$ satisfies $\varphi(t)>0$ for all $t>0$. Then $T$ has a unique fixed point $x^{*} \in X$, and $\left\{T^{n} x_{0}\right\}$ converges to $x^{*}$ for each $x_{0} \in X$. 
Proof Take $x_{0} \in X$ arbitrarily and define the sequence $\left\{x_{n}\right\}$ by $x_{n}=T x_{n-1}$ for each $n \in \mathbb{N}$. Since $T$ is a probabilistic $\varphi$-contraction, we have

$$
F_{x_{n}, x_{m}}(\varphi(t))=F_{T x_{n-1}, T x_{m-1}}(\varphi(t)) \geq F_{x_{n-1}, x_{m-1}}(t), \quad \forall m, n \in \mathbb{N} \text { and } \forall t>0 .
$$

So, from Lemma 3.4 it follows that $\left\{x_{n}\right\}$ is a Cauchy sequence. Since $X$ is complete, there exists $x^{*} \in X$ such that $x_{n} \rightarrow x^{*}$ as $n \rightarrow \infty$.

Next we show that $x^{*}$ is a fixed point of $T$. For any $t>0$, Lemma 3.1 shows that there exists $r \geq t$ such that $\varphi(r)<t$. By the monotonicity of $\Delta$ we get

$$
\begin{aligned}
F_{x^{*}, T x^{*}}(t) & \geq \Delta\left(F_{x^{*}, x_{n+1}}(t-\varphi(r)), F_{x_{n+1}, T x^{*}}(\varphi(r))\right) \\
& =\Delta\left(F_{x^{*}, x_{n+1}}(t-\varphi(r)), F_{T x_{n}, T x^{*}}(\varphi(r))\right) \\
& \geq \Delta\left(F_{x^{*}, x_{n+1}}(t-\varphi(r)), F_{x_{n}, x^{*}}(r)\right) \\
& \geq \Delta\left(c_{n}, c_{n}\right),
\end{aligned}
$$

where $c_{n}=\min \left\{F_{x^{*}, x_{n+1}}(t-\varphi(r)), F_{x_{n}, x^{*}}(r)\right\}$. Since $c_{n} \rightarrow 1$ as $n \rightarrow \infty$ and $\Delta$ is continuous at $(1,1)$, from (3.10) we have

$$
F_{x^{*}, T x^{*}}(t) \geq \Delta\left(c_{n}, c_{n}\right) \rightarrow \Delta(1,1)=1,
$$

which implies that $x^{*}=T x^{*}$.

Finally, we prove that $x^{*}$ is the unique fixed point of $T$. Suppose that $T$ has another fixed point $x^{\prime} \in X$. Then we have

$$
F_{x^{*}, x^{\prime}}(\varphi(t))=F_{T x^{*}, T x^{\prime}}(\varphi(t)) \geq F_{x^{*}, x^{\prime}}(t), \quad \forall t>0 .
$$

From Lemma 3.2 it follows that $x^{*}=x^{\prime}$. Thus $x^{*}$ is the unique fixed point of $T$. This completes the proof.

Corollary 3.1 Let $(X, F, \Delta)$ be a complete Menger space where $\Delta$ is of H-type and continuous at $(1,1)$. Let $T_{0}, T_{1}: X \rightarrow X$ be two mappings such that

$$
F_{T_{0} x, T_{0} y}(\varphi(t)) \geq F_{x, y}(t) \quad \text { and } \quad F_{T_{1} x, T_{1} y}(t) \geq F_{x, y}(t) \quad \text { for all } x, y \in X \text { and } t>0,
$$

where $\varphi \in \boldsymbol{\Phi}_{\mathbf{w}^{*}}$ satisfies $\varphi(t)>0$ for all $t>0$. If $T_{0}$ commutes with $T_{1}$, then $T_{0}$ and $T_{1}$ have a unique common fixed point in $X$.

Proof Let $T=T_{0} T_{1}$. Then (3.11) implies that $T$ is a probabilistic $\varphi$-contraction. From Theorem 3.1 it follows that $T$ has a unique fixed point $x^{*} \in X$. Since $T_{0}$ commutes with $T_{1}$, we have $T_{0} T_{1} x^{*}=T_{1} T_{0} x^{*}$. Further we have $T\left(T_{0} x^{*}\right)=\left(T_{0} T_{1}\right)\left(T_{0} x^{*}\right)=T_{0}\left(T_{0} T_{1} x^{*}\right)=T_{0}\left(T x^{*}\right)=$ $T_{0} x^{*}$, which implies that $T_{0} x^{*}$ is a fixed point of $T$. Since $T$ has a unique fixed point $x^{*}$, one has $T_{0} x^{*}=x^{*}$. Similarly, we have $T_{1} x^{*}=x^{*}$. Thus $x^{*}$ is the common fixed point of $T_{0}$ and $T_{1}$. Assume that $x^{\prime} \in X$ is another common fixed point of $T_{0}$ and $T_{1}$. Since $T_{0}$ commutes with $T_{1}$, we have $T\left(T_{0} x^{\prime}\right)=\left(T_{0} T_{1}\right)\left(T_{0} x^{\prime}\right)=T_{0}\left(T_{0} T_{1} x^{\prime}\right)=T_{0}\left(T_{1} T_{0} x^{\prime}\right)=T_{0} x^{\prime}$, which implies that $T_{0} x^{\prime}$ is the fixed point of $T$. Since $x^{*}$ is a unique fixed point of $T$, one has $x^{\prime}=T_{0} x^{\prime}=x^{*}$. Thus $x^{*}$ is the unique common fixed point of $T_{0}$ and $T_{1}$. This completes the proof. 
Finally, we give an example to illustrate Theorem 3.1.

Example 3.2 Let $X=\left\{3^{n+2}: n \in \mathbb{N}\right\} \cup\{0,3\}$ and define the mapping $F: X \times X \rightarrow \mathcal{D}^{+}$by $F_{x, y}(0)=0$ for all $x, y \in X, F_{x, x}(t)=1$ for all $x \in X$ and $t>0$,

$$
F_{0,3}(t)=F_{3,0}(t)=\left\{\begin{array}{ll}
\frac{3}{5}, & 0<t \leq 3, \\
1, & t>3
\end{array} \quad \text { and } \quad F_{x, y}(t)=F_{y, x}(t)= \begin{cases}\frac{1}{2}, & 0<t \leq|x-y| \\
1, & t>|x-y|\end{cases}\right.
$$

for all $x, y \in X$ with $x \neq y$ and $\{x, y\} \neq\{0,3\}$. It is easy to see that $\left(X, F, \Delta_{M}\right)$ is a complete Menger space.

Let $T: X \rightarrow X$ be a mapping defined by $T 0=T 3=T 27=0$ and $T 3^{n+3}=3^{n+2}$ for each $n \in \mathbb{N}$. Let $\varphi: \mathbb{R}^{+} \rightarrow \mathbb{R}^{+}$be a function defined by

$$
\varphi(t)= \begin{cases}t, & \text { if } 0 \leq t \leq 1 \\ t-1, & \text { if } t>1\end{cases}
$$

Then $\varphi \in \boldsymbol{\Phi}_{\mathbf{w}^{*}}$, but $\varphi \notin \boldsymbol{\Phi}_{\mathbf{w}}$; see Example 3.1.

Next we show that $T$ is a probabilistic $\varphi$-contraction, i.e., $T$ satisfies the following condition:

$$
F_{T x, T y}(\varphi(t)) \geq F_{x, y}(t) \quad \text { for all } x, y \in X \text { and } t>0 .
$$

First, it is easy to see that for $x, y \in\{0,3,27\}$, (3.12) holds for all $t>0$ since $T 0=T 3=$ $T 27=0$. Next we show that (3.12) holds for all $x, y \in X$ with $x \neq y$ and $\{x, y\} \nsubseteq\{0,3,27\}$ and $t>0$. Obviously, if $|T x-T y|<\varphi(t)$, then $F_{T x, T y}(\varphi(t))=1 \geq F_{x, y}(t)$. So (3.12) holds. Now we consider all $x, y \in X$ with $x \neq y$ and $\{x, y\} \nsubseteq\{0,3,27\}$ and $t>0$ with $|T x-T y| \geq \varphi(t)$ by the following cases:

(a) For $(x, y) \in\left\{\left(0,3^{n+3}\right),\left(3,3^{n+3}\right),\left(27,3^{n+3}\right): n \in \mathbb{N}\right\}$, it is easy to conclude that $\varphi(t) \leq|T x-T y|$ implies that $t \leq|x-y|$ for all $t>0$. Thus if $\varphi(t) \leq|T x-T y|$, then

$$
F_{T x, T y}(\varphi(t))=\frac{1}{2}=F_{x, y}(t) \quad \text { for all } t>0 .
$$

Therefore (3.12) holds.

(b) For $(x, y) \in\left\{\left(3^{n+3}, 3^{m+3}\right): m, n \in \mathbb{N}\right.$ with $\left.m>n\right\}$, we have

$\varphi(t) \leq|T x-T y|=3^{m+2}-3^{n+2}<3\left(3^{m+2}-3^{n+2}\right)=|y-x|$ for $t \in(0,1]$. For $t>1$, from

$\varphi(t)=t-1 \leq|T x-T y|=3^{m+2}-3^{n+2}$, we have

$t \leq 3^{m+2}-3^{n+2}+1<3^{m+3}-3^{n+3}=|x-y|$ since

$3^{m+3}-3^{n+3}-3^{m+2}+3^{n+2}=2\left(3^{m+2}-3^{n+2}\right)>1$. So $\varphi(t) \leq|T x-T y|$ implies that

$t \leq|x-y|$ for all $t>0$. Thus if $\varphi(t) \leq|T x-T y|$, then

$$
F_{T x, T y}(\varphi(t))=\frac{1}{2}=F_{x, y}(t) \quad \text { for all } t>0 .
$$

Therefore (3.12) holds.

By the discussion above, (3.12) holds for all $x, y \in X$ and $t>0$. Therefore, $T$ is a probabilistic $\varphi$-contraction. All the conditions of Theorem 3.1 are satisfied. By Theorem 3.1, $T$ has a unique fixed point $x^{*} \in X$. Obviously, $x^{*}=0$ is the unique fixed point of $T$. However, since $\varphi \notin \boldsymbol{\Phi}_{\mathbf{w}}$, Theorem 1.2, i.e., Theorem 3.1 of [14] cannot be applied to this example. 


\section{Conclusion}

In this paper, we prove a new fixed point theorems for a probabilistic $\varphi$-contraction in Menger spaces. In the theorem, a more weak condition on the gauge function $\varphi$ is required. Thus our result improves Theorem 1.2 of Fang [14] and some others, such as Jachymski [13], Ćirić [2], and Xiao et al. [19]. By using Theorem 3.1, it is easy to prove some fixed point theorems for $\varphi$-contraction in fuzzy metric spaces like Theorems 4.1-4.4 in [14]. For shortening the length of this paper, we omit the proofs of these theorems.

Competing interests

The authors declare that they have no competing interests.

\section{Authors' contributions}

All authors read and approved the final manuscript.

\section{Author details}

${ }^{1}$ Department of Mathematics and Physics, North China Electric Power University, Baoding, 071003, China. ${ }^{2}$ Department of Mathematics and Sciences, Shijiazhuang University of Economics, Shijiazhuang, 050031, China.

\section{Acknowledgements}

This work is supported by the Fundamental Research Funds for the Central Universities (Grant numbers: 13MS109, 2014MS164, 2014ZD44, 2015MS78). The authors thank the editor and reviewers.

Received: 10 April 2015 Accepted: 4 September 2015 Published online: 06 November 2015

\section{References}

1. Sehgal, VM, Bharucha-Reid, AT: Fixed points of contraction mappings on PM-spaces. Math. Syst. Theory 6, 97-102 (1972)

2. Ćirić, L: Solving the Banach fixed point principle for nonlinear contractions in probabilistic metric spaces. Nonlinear Anal. 72, 2009-2018 (2010)

3. Duh, MF, Huang, YY: Common fixed points for mappings on complete Menger spaces. Far East J. Math. Sci. 6, 169-193 (1998)

4. Fang, JX: A note on fixed point theorems of Hadžić. Fuzzy Sets Syst. 48, 391-395 (1992)

5. Fang, JX: Common fixed point theorems of compatible and weakly compatible maps in Menger spaces. Nonlinear Anal. 71, 1833-1843 (2009)

6. O'Regan, D, Saadati, R: Nonlinear contraction theorems in probabilistic spaces. Appl. Math. Comput. 195, 86-93 (2008)

7. Xiao, JZ, Zhu, XH, Cao, YF: Common coupled fixed point results for probabilistic $\varphi$-contractions in Menger spaces. Nonlinear Anal., Theory Methods Appl. 74, 4589-4600 (2011)

8. Chauhan, S, Pant, BD: Fixed point theorems for compatible and subsequentially continuous mappings in Menger spaces. J. Nonlinear Sci. Appl. 7, 78-89 (2014)

9. Mihet, D: Common coupled fixed point theorems for contractive mappings in fuzzy metric spaces. J. Nonlinear Sci. Appl. 6, 35-40 (2013)

10. Phiangsungnoen, $S$, Kumam, P: Fuzzy fixed point theorems for multivalued fuzzy contractions in $b$-metric spaces. J. Nonlinear Sci. Appl. 8, 55-63 (2015)

11. Chauhan, S, Shatanawi, W, Kumar, S, Radenovic, S: Existence and uniqueness of fixed points in modified intuitionistic fuzzy metric spaces. J. Nonlinear Sci. Appl. 7, 28-41 (2014)

12. $\mathrm{Xu}, \mathrm{WQ}, \mathrm{Zhu}, \mathrm{CX}, \mathrm{Wu}, \mathrm{ZQ}, \mathrm{Zhu}, \mathrm{L}$ : Fixed point theorems for two new types of cyclic weakly contractive mappings in partially ordered Menger PM-spaces. J. Nonlinear Sci. Appl. 8, 412-422 (2015)

13. Jachymski, J: On probabilistic $\varphi$-contractions on Menger spaces. Nonlinear Anal. 73, 2199-2203 (2010)

14. Fang, JX: On $\varphi$-contractions in probabilistic and fuzzy metric spaces. Fuzzy Sets Syst. 267, $86-99$ (2015)

15. Schweizer, B, Sklar, A, Thop, E: The metrization of statistical metric spaces. Pac. J. Math. 10, 673-675 (1960)

16. Hadžić, O, Pap, E: Fixed Point Theory in Probabilistic Metric Spaces. Mathematics and Its Application, vol. 536. Kluwer Academic, Dordrecht (2001)

17. Roldán, A, Martínez-Moreno, J, Roldán, C: Tripled fixed point theorem in fuzzy metric spaces and applications. Fixed Point Theory Appl. 2013, Article ID 29 (2013)

18. Menger, K: Statistical metrics. Proc. Natl. Acad. Sci. USA 28, 535-537 (1942)

19. Xiao, JZ, Zhu, XH, Liu, XY: An alternative characterization of probabilistic Menger spaces with $H$-type triangular norms. Fuzzy Sets Syst. 227, 107-114 (2013) 